\title{
Some remarks on well-posedness of the higher-dimensional chemorepulsion system \\ by
}

\author{
Tomasz CIEŚLAK and Kentarou FUJIE
}

Presented by Piotr BILER

Summary. We consider a fully parabolic system of chemorepulsion in higher dimensions. We introduce a new energy-like identity and comment on its relation to the $\mathrm{Li}-\mathrm{Yau}-$ Hamilton inequalities. Next, we show that for the chemorepellent moving much more slowly than the cells, the time global well-posedness holds.

1. Introduction. Let $\Omega$ be a bounded subdomain of $\mathbb{R}^{n}$ with a regular boundary, and let $u$ and $v$ be real valued functions. We consider the following fully parabolic chemorepulsion system:

$$
\begin{cases}\partial_{t} u=\nabla \cdot(\nabla u+u \nabla v) & \text { in }(0, \infty) \times \Omega, \\ \partial_{t} v=\Delta v-v+u & \text { in }(0, \infty) \times \Omega, \\ u(0, x)=u_{0}(x) \geq 0, \quad v(0, x)=v_{0}(x) \geq 0, & \text { for } x \in \Omega .\end{cases}
$$

We impose the boundary conditions

$$
\frac{\partial u}{\partial \vec{n}}=\frac{\partial v}{\partial \vec{n}}=0 \quad \text { on }(0, \infty) \times \partial \Omega .
$$

Such a system can be considered as a version of repulsive action of a substance $v$ on cells $u$, describing an opposite phenomenon to the well-known aggregation mechanism described by the Keller-Segel system. The only difference from the Keller-Segel model is the sign in the first equation of (1.1).

Due to the difference in sign, one expects a completely different behaviour of solutions. Namely, instead of collapsing, the density of cells $u$ is

2010 Mathematics Subject Classification: 35B45, 35K45, 92C17.

Key words and phrases: boundedness of solutions, chemotaxis, Lyapunov-like functional. Received 24 March 2019.

Published online 14 June 2019. 
expected to stay bounded and tend to flat steady states. Indeed, this is the case in space dimension 2 (see [4]). Moreover, in higher dimensions global weak solutions are known, but it is not proven (though expected) that they are unique and bounded. The main device used in the proofs in [4] is the Lyapunov functional satisfying

$$
\begin{aligned}
\frac{d}{d t}\left(\int_{\Omega} u \log u d x+\frac{1}{2} \int_{\Omega}|\nabla v|^{2} d x\right) \\
\quad=-\int_{\Omega}|\nabla \sqrt{u}|^{2} d x-\int_{\Omega}|\Delta v|^{2} d x-\int_{\Omega}|\nabla v|^{2} d x .
\end{aligned}
$$

It gives enough information to prove two-dimensional boundedness (and convergence to steady states), but it is not useful in the problem of wellposedness of regular solutions in higher dimensions. Weak time global solutions can also be constructed in higher dimensions (see [4] or [3]), but regularity (even the bound in $L^{\infty}$ ) of such solutions is not known, though it is expected.

Since [4] there have been a lot of studies on the topic, on the one hand since such a system appears as part of a model describing the competition between attraction and repulsion in cells biology (see [14]), on the other hand since the problem is quite natural and simple energy estimates do not lead to the expected result. Many authors considered the attraction vs. repulsion problem (see for instance [14, [7] or [11]), but usually in their studies they used either the functional (1.2) or some more complicated functionals related to the more complicated structure of the full attraction-repulsion system (see [11] or [7]). However, no essential progress on (1.1) has been made. The latter would clearly advance the study of attraction-repulsion competition. Actually, some nonlinear versions of (1.1) have also been studied (see [5, 13]), with well-posedness results weaker (for stronger diffusion or weaker sensitivity) than those in [4]. The methods were based on extensions of $(1.2)$ to the nonlinear diffusion/sensitivity setting. The problem of global well-posedness of regular solutions of (1.1) remains challenging in dimensions $n \geq 3$.

In the present note we introduce a new energy-like identity obtained recently. However, we have been unable to utilize it to get global well-posedness of classical solutions to (1.1). All the same it seems interesting, exhibiting some connections to the Li-Yau inequality. On the other hand, in the second part of the paper we prove the global existence of solutions for 1.1) with the speed of the cells being small compared to the speed of the repellent. To this end we imitate the method of [6]. Local existence results in [4] ensure that the unique positive (for $t \in\left(0, T_{\max }\right)$ ) mass conserving regular solutions to (1.1) exist, and moreover they are global provided

$$
\sup _{t \in\left(0, T_{\max }\right)}\|u(t, \cdot)\|_{L^{\infty}(\Omega)}<\infty .
$$


2. A new energy-like functional. In this section we introduce a new energy-type identity and then comment on its possible application to the study of global existence for (1.1), provided some sort of Li-Yau type inequality holds for the system of parabolic equations (1.1). So far, Li-Yau type inequalities seem to be known only for single equations. Moreover, the maximum principle seems to be essential to the proof.

If we consider the first equation of (1.1) as a continuity equation of density $u$ with a velocity field given by $V$ (see (2.1)), then by the hydrodynamic analogy, one would like to trace the time evolution of $\int_{\Omega} u V^{2}$, playing the role of kinetic energy. We see that the following identity holds.

TheOREM 2.1. Let $(u, v)$ be a solution of (1.1). Define

$$
V:=\frac{\nabla u}{u}+\nabla v
$$

Then

$$
\begin{array}{r}
\frac{d}{d t}\left(\int_{\Omega} u|V|^{2} d x+\int_{\Omega} v_{t}^{2} d x\right)+2 \int_{\Omega}\left|\nabla v_{t}\right|^{2} d x+2 \int_{\Omega}\left|v_{t}\right|^{2} d x+2 \int_{\Omega} \frac{u_{t}^{2}}{u} d x \\
=-2 \int_{\Omega} u\left(V^{T}\right) D^{2}(\log u+v)(V),
\end{array}
$$

where $x^{T}$ denotes the transpose of the vector $x$ and $D^{2}(f)$ denotes the matrix of second derivatives of the function $f$.

Proof. We start by computing the time derivative

$$
\frac{d}{d t} \int_{\Omega} u|V|^{2} d x=\int_{\Omega} u_{t}|V|^{2} d x+2 \int_{\Omega} u V V_{t} d x .
$$

Next we notice that

$$
\begin{aligned}
2 \int_{\Omega} u V \cdot & V_{t} d x=2 \int_{\Omega} u\left(\frac{\nabla u}{u}+\nabla v\right) \cdot \nabla(\log u+v)_{t} d x \\
= & -2 \int_{\Omega} \nabla u \cdot\left(\frac{\nabla u}{u}+\nabla v\right)(\log u+v)_{t} d x \\
& -2 \int_{\Omega} u \nabla \cdot\left(\frac{\nabla u}{u}+\nabla v\right)(\log u+v)_{t} d x \\
= & -2 \int_{\Omega}\left(\nabla u \cdot\left(\frac{\nabla u}{u}+\nabla v\right) d x+u \nabla \cdot\left(\frac{\nabla u}{u}+\nabla v\right)\right) \frac{u_{t}}{u} d x \\
& -2 \int_{\Omega}\left(\nabla u \cdot\left(\frac{\nabla u}{u}+\nabla v\right)+u \nabla \cdot\left(\frac{\nabla u}{u}+\nabla v\right)\right) v_{t} d x \\
= & -2 \int_{\Omega} \frac{u_{t}^{2}}{u} d x-2 \int_{\Omega} u_{t} v_{t} d x .
\end{aligned}
$$


Differentiating the second equation of 11.1 with respect to time and next multiplying it by $v_{t}$ we arrive at

$$
\frac{d}{d t} \int_{\Omega} v_{t}^{2} d x=2 \int_{\Omega} v_{t t} v_{t} d x=-2 \int_{\Omega}\left|\nabla v_{t}\right|^{2} d x-2 \int_{\Omega} v_{t}^{2} d x+2 \int_{\Omega} u_{t} v_{t} d x .
$$

Plugging (2.4) and $(2.5)$ into 2.3$)$ we arrive at

$$
\begin{aligned}
& \frac{d}{d t} \int_{\Omega} u|V|^{2} d x \\
= & \int_{\Omega} u_{t}|V|^{2} d x-\frac{d}{d t} \int_{\Omega} v_{t}^{2} d x-2 \int_{\Omega} \frac{u_{t}^{2}}{u} d x-2 \int_{\Omega}\left|\nabla v_{t}\right|^{2} d x-2 \int_{\Omega} v_{t}^{2} d x,
\end{aligned}
$$

so (2.2) follows from the identity

$$
\begin{aligned}
\int_{\Omega} u_{t}|V|^{2} d x & =\int_{\Omega} \nabla \cdot(u \nabla(\log u+v))|\nabla(\log u+v)|^{2} d x \\
& =-2 \int_{\Omega} u(\nabla(\log u+v))^{T} D^{2}(\log u+v) \nabla(\log u+v) d x .
\end{aligned}
$$

REMARK 2.2. Note that in the course of the proof, we have obtained the following interesting identity (see (2.6)):

$$
\begin{aligned}
\frac{d}{d t} \int_{\Omega} u|V|^{2} d x+\frac{d}{d t} \int_{\Omega} v_{t}^{2} d x+2 \int_{\Omega} \frac{u_{t}^{2}}{u} d x+2 \int_{\Omega}\left|\nabla v_{t}\right|^{2} d x & +2 \int_{\Omega} v_{t}^{2} d x \\
& =\int_{\Omega} u_{t}|V|^{2} d x .
\end{aligned}
$$

REMARK 2.3. We point out that a bound from below on the second space derivatives of $\log u+v$, such as the one for the heat equation (see [8]), would enable us to use (2.2) to deduce a bound on $\int_{\Omega} v_{t}^{2} d x$. Indeed, if

$$
(\nabla(\log u+v))^{T} D^{2}(\log u+v) \nabla(\log u+v) \geq-c_{0}|\nabla(\log u+v)|^{2}
$$

for some nonnegative constant $c_{0}$, the pair $(u, v)$ being a solution to (1.1) (notice that one can start from $C^{2}$ initial data so that initially (for $\left(u_{0}, v_{0}\right)$ ) such an estimate holds for some $c_{0}$ ), then integration of (2.2) and a Gronwall type argument lead to an estimate of $\int_{\Omega} v_{t}^{2} d x$.

As already mentioned, however, Li-Yau-Hamilton type inequalities are only known to hold for single equations; see [8] for the heat equation or [2, 1] for the equation

$$
u_{t}=\Delta u+\nabla u \cdot \nabla h,
$$

where $h$ is a given convex potential. The question is whether such an estimate can also hold for the system 1.1). 
3. Well-posedness for large speed of cells. In this section we focus on the global existence of regular solutions of

$$
\begin{cases}\tau \partial_{t} u=\nabla \cdot(\nabla u+u \nabla v) & \text { in }(0, \infty) \times \Omega, \\ \partial_{t} v=\Delta v-v+u & \text { in }(0, \infty) \times \Omega,\end{cases}
$$

for $\tau$ small enough, under zero Neumann data and for nonnegative initial data $u_{0}, v_{0}$. In dimensions higher than 2 nothing is known concerning the global well-posedness of solutions to the fully parabolic problem. However, an easy energy estimate leads in a standard way to the global existence result for the parabolic-elliptic problem (where instead of $v_{t}$ in the second equation of (3.1) one puts zero). For the rigorous proof one should consult [12, where a slightly more general problem is studied. In what follows we focus on an opposite problem and study the neighbourhood of the ellipticparabolic problem, meaning (3.1) with $\tau$ small (or even zero). We follow the argument in [6].

Proposition 3.1. Let $n \geq 2$ and let $\Omega$ be a bounded domain in $\mathbb{R}^{n}$. Suppose $\tau=0, \int_{\Omega} u(x, t) d x=\lambda$ with $\lambda>0$ and the initial data $u_{0} \in$ $W^{1, \infty}(\Omega)$ is nonnegative. Then the solution of (3.1) exists globally in time and remains bounded.

Proof. Taking $\tau=0$, we have

$$
0=\nabla \cdot(u \nabla(\log u+v)),
$$

thus $\log u+v=$ const, and hence by the constraint on $u$,

$$
u=\lambda \frac{\exp (-v)}{\int_{\Omega} \exp (-v) d x} .
$$

Therefore the system $(3.1)$ is changed to the single heat equation with nonlinear nonlocal term:

$$
v_{t}=\Delta v-v+\lambda \frac{\exp (-v)}{\int_{\Omega} \exp (-v) d x} .
$$

Since the nonlocal term on the right-hand side can be estimated as in (3.3) below and due to fact that $e^{-v} \leq 1$, the parabolic regularity estimate gives the claim.

The above proposition can be extended to the system (3.1) with small positive $\tau$.

THEOREM 3.2. Let $n \geq 2$ and let $\Omega$ be a bounded convex domain in $\mathbb{R}^{n}$. Suppose that the initial data $\left(u_{0}, v_{0}\right) \in C^{0}(\bar{\Omega}) \times W_{N}^{2, q}(\Omega)$ are nonnegative with some $q>n$. Then the solution of 3.1 exists globally in time and remains bounded if $\tau$ is sufficiently small. 
The proof of Theorem 3.2 splits into several steps. Proceeding as in 6 , Lemma 3.2], we obtain a lower estimate of the maximal existence time of the solution to (3.1), independent of $\tau>0$.

LEMma 3.3. Let $\left(u_{\tau}, v_{\tau}\right)$ be the solution to 3.1) with $\tau \in(0,1]$ in $\left(0, T_{\max }(\tau)\right) \times \Omega$. There are positive constants $T_{\min }>0$ and $L>0$ such that

$$
\begin{aligned}
T_{\max }(\tau)>T_{\min } & \text { for } \tau \in(0,1], \\
\left\|\left(u_{\tau}, v_{\tau}\right)\right\|_{L^{\infty}\left(\left(0, T_{\min }\right) \times \bar{\Omega}\right)} \leq L & \text { for } \tau \in(0,1], \\
\left\|\left(u_{\tau}, v_{\tau}\right)\right\|_{C^{2,1}\left(\left(0, T_{\min }\right) \times \bar{\Omega}\right)}<\infty & \text { for } \tau \in(0,1] .
\end{aligned}
$$

Here $T_{\max }(\tau)$ is the maximal existence time of the solutions to (3.1), and $T_{\min }>0$ and $L>0$ are independent of $\tau$.

For $\tau \in(0,1]$, let $\left(u_{\tau}, v_{\tau}\right)$ be a solution to $(3.1)$ in $\left(0, T_{\max }(\tau)\right) \times \Omega$, where $T_{\max }(\tau)$ is the maximal existence time of the classical solution. Put

$$
z_{\tau}=\frac{\exp \left(-v_{\tau}\right)}{\int_{\Omega} \exp \left(-v_{\tau}\right) d x} \quad \text { and } \quad w_{\tau}=\frac{u_{\tau}}{z_{\tau}} .
$$

Then $v_{\tau}$ and $w_{\tau}$ satisfy

$$
\frac{\partial v_{\tau}}{\partial t}=\Delta v_{\tau}-v_{\tau}+w_{\tau} \frac{\exp \left(-v_{\tau}\right)}{\int_{\Omega} \exp \left(-v_{\tau}\right) d x} .
$$

Here we can estimate

$$
\frac{\|\exp (-v(s))\|_{L^{\infty}(\Omega)}}{\int_{\Omega} \exp (-v(s)) d x} \leq \frac{1}{\int_{\Omega} \exp (-v(s)) d x} .
$$

Jensen's inequality implies that for $s>0$,

$$
\begin{aligned}
\int_{\Omega} \exp (-v(s, x)) d x & =|\Omega| \int_{\Omega} \exp (-v(s, x)) \frac{d x}{|\Omega|} \\
& \geq|\Omega| \exp \left(-\int_{\Omega} v(s, x) \frac{d x}{|\Omega|}\right) \\
& \geq|\Omega| \exp \left(-\frac{1}{|\Omega|} \max _{s>0} \int_{\Omega} v(s, x) d x\right) .
\end{aligned}
$$

Integrating the second equation of (3.1), we arrive at

$$
\max _{s>0} \int_{\Omega} v(s, x) d x \leq \max \left\{\int_{\Omega} u_{0} d x, \int_{\Omega} v_{0} d x\right\} .
$$

Therefore

$$
\left\|\frac{\exp (-v(s))}{\int_{\Omega} \exp (-v(s)) d x}\right\|_{L^{\infty}(\Omega)} \leq C\left(u_{0}, v_{0}\right)
$$


Proposition 3.4. There exists $H_{1}>0$ such that for all $\tau \in(0,1]$,

$$
\left\|w_{\tau}(t)\right\|_{L^{\infty}(\Omega)}<H_{1} \quad \text { for } t \in\left[0, T_{\min }\right],
$$

where $T_{\min }$ is the constant in Lemma 3.3 .

Proof. By the definition we estimate

$$
\left\|w_{\tau}\right\|_{L^{\infty}(\Omega)}=\left\|\frac{u_{\tau}}{z_{\tau}}\right\|_{L^{\infty}(\Omega)} \leq\left\|u_{\tau}\right\|_{L^{\infty}(\Omega)}\left\|\frac{\int_{\Omega} \exp \left(-v_{\tau}\right) d x}{\exp \left(-v_{\tau}\right)}\right\|_{L^{\infty}(\Omega)} .
$$

Lemma 3.3 yields

$$
\left\|w_{\tau}\right\|_{L^{\infty}(\Omega)} \leq L \exp (L)|\Omega|,
$$

where $L>0$ is the constant in Lemma 3.3

Let $H:=\max \left\{2\|w(0)\|_{L^{\infty}(\Omega)}, H_{1}\right\}$. We define

$$
\begin{aligned}
S_{2}(\tau) & :=\sup \left\{T \in\left(0, T_{\max }(\tau)\right):\left\|w_{\tau}(t)\right\|_{L^{\infty}(\Omega)} \leq H \text { for } t \in[0, T)\right\} \\
& \geq T_{\min }>0 .
\end{aligned}
$$

Henceforth, for simplicity, we omit the index $\tau$. Our strategy is to prove $S_{2}(\tau)=\infty$ for sufficiently small $\tau>0$. Since $v$ satisfies $(3.2)$ below and due to the estimate (3.4), parabolic regularity yields the following lemma.

Lemma 3.5. There exists a positive constant $C(H)$ depending only on $H$ and independent of $\tau>0$ such that

$$
\|v(t)\|_{L^{\infty}(\Omega)} \leq 2\left\|v_{0}\right\|_{L^{\infty}(\Omega)}+C(H)=: v^{*}(H) \quad \text { for } t \in\left(0, S_{2}(\tau)\right) .
$$

Moreover, there exists a positive constant $C\left(H, v_{0}\right)$ depending on $H$ and $v_{0}$ and independent of $\tau>0$ such that for all $p \in[1, \infty)$,

$$
\|\nabla v(t)\|_{L^{\infty}(\Omega)}+\|\nabla v(t)\|_{L^{p}(\Omega)} \leq C\left(H, v_{0}\right) \quad \text { for } t \in\left(0, S_{2}(\tau)\right) .
$$

For $T \in\left(0, T_{\min }\right)$, take an integer $J$ such that $(J-1) T / 2<S_{2}(\tau) \leq$ $J T / 2$. Let

$$
\begin{aligned}
& T_{j}= \begin{cases}j T / 2 & \text { for } j=0,1, \ldots, J-1, \\
S_{2}(\tau) & \text { for } j=J,\end{cases} \\
& z_{j}=z\left(\cdot, T_{j}\right), \quad v_{j}=v\left(\cdot, T_{j}\right) .
\end{aligned}
$$

and let $\mathcal{A}_{j}=\mathcal{A}\left(z_{j}\right)$, where the operator $\mathcal{A}$ is defined as

$$
\mathcal{A}=\mathcal{A}(z)=\frac{1}{z} \nabla \cdot(z \nabla) .
$$

Since the first equation in $(3.1)$ implies

$$
\begin{aligned}
\frac{\tau}{z} \frac{\partial u}{\partial t} & =\frac{1}{z}(\Delta u+\nabla \cdot(u \nabla v))=\frac{1}{z}(\Delta(w z)-\nabla \cdot(w \nabla z)) \\
& =\frac{1}{z} \nabla \cdot(z \nabla w),
\end{aligned}
$$


we conclude that for $j=0,1, \ldots, J-1$, the pair of functions $(v, w)$ satisfies

$$
\begin{cases}\tau \frac{\partial w}{\partial t}=\mathcal{A}_{j} w+\nabla \log \frac{z}{z_{j}} \cdot \nabla w-\tau \frac{\partial \log z}{\partial t} w & \text { in }\left(T_{j}, T_{j+1}\right) \times \Omega, \\ \frac{\partial v}{\partial t}=\Delta v-v+w \frac{\exp (-v)}{\int_{\Omega} \exp (-v) d x} & \text { in }\left(T_{j}, T_{j+1}\right) \times \Omega, \\ \frac{\partial w}{\partial \nu}=\frac{\partial v}{\partial \nu}=0 & \text { on }\left(T_{j}, T_{j+1}\right) \times \partial \Omega .\end{cases}
$$

In the following lemmas we give estimates of $\nabla \log \left(z / z_{j}\right)$ and $\frac{\partial z}{\partial s}$ in $\left(0, S_{2}(t)\right) \times \Omega$.

Lemma 3.6. Assume that $\alpha, \beta \in(0,1)$ and $q>n$ satisfy $1+\alpha+n / q<2 \beta$. Then, for $j=0,1, \ldots, J-1$ and $t \in\left[T_{j}, T_{j+1}\right]$,

$$
\left\|\nabla \log \left(\frac{z(t)}{z\left(T_{j}\right)}\right)\right\|_{L^{\infty}(\Omega)} \leq C\left(H, v_{0}\right) T^{\alpha / 2},
$$

where $C\left(H, v_{0}\right)$ is a positive constant depending on $v_{0}$ and $H$, and independent of $\tau>0$.

Proof. From $\nabla \log z=-\nabla v$ and $\nabla \log z_{i}=-\nabla v_{i}$ we see that

$$
\left|\nabla \log \frac{z(t)}{z_{j}}\right|=\left|\nabla\left(v(t)-v_{j}\right)\right| .
$$

Now [6, Lemma 2.3(v)] with $1+\alpha+n / q<2 \beta$ ensures that

$$
\begin{aligned}
& \left\|\nabla v(t)-\nabla v_{j}\right\|_{L^{\infty}(\Omega)} \\
& \quad \leq C T^{\alpha / 2}\left(\left\|\left(\Delta_{N}-1\right)^{\beta} v_{j}\right\|_{L^{q}(\Omega)}+H \sup _{t \in\left(T_{j}, T_{j+1}\right)}\left\|\frac{\exp (-v(t))}{\int_{\Omega} \exp (-v(t)) d x}\right\|_{L^{q}(\Omega)}\right)
\end{aligned}
$$

for all $t \in\left[t_{j}, T_{j+1}\right]$. Since [6, Lemma 2.3(iii)] implies that

$$
\left\|\left(\Delta_{N}-1\right)^{\beta} v_{j}\right\|_{L^{q}(\Omega)} \leq C\left(H, v_{0}\right)
$$

with some $C\left(H, v_{0}\right)>0$, we deduce from this inequality, (3.4) and Lemma 3.5 that

$$
\begin{aligned}
\left\|\nabla v(t)-\nabla v_{j}\right\|_{L^{\infty}(\Omega)} & \leq C T^{\alpha / 2}\left(\left\|\left(\Delta_{N}-1\right)^{\beta} v_{j}\right\|_{L^{q}(\Omega)}+C\left(u_{0}, v_{0}\right)\right) \\
& \leq C\left(H, v_{0}\right) T^{\alpha / 2} .
\end{aligned}
$$

Thus by (3.7), there exists a positive constant $C\left(H, v_{0}\right)$ such that

$$
\left\|\nabla \log \frac{z(t)}{z_{j}}\right\|_{L^{\infty}(\Omega)} \leq C\left(H, v_{0}\right) T^{\alpha / 2} \quad \text { for } t \in\left[T_{j}, T_{j+1}\right] .
$$


Lemma 3.7. Let $q, p \in(1, \infty)$. Then there exists a positive constant $C$ depending on $H, v_{0}, p$ and $q$ such that

$$
\int_{0}^{t} e^{s-t}\left\|\frac{\partial \log z}{\partial s}\right\|_{L^{q}(\Omega)}^{p} d s \leq C\left(t e^{-t}\left\|v_{0}\right\|_{W^{2, q}(\Omega)}^{p}+1\right) \quad \text { for } t \in\left(0, S_{2}(\tau)\right) .
$$

Proof. Since

$$
\left(e^{t / p} v\right)_{t}=\Delta\left(e^{t / p} v\right)-e^{t / p} v+\frac{1}{p}\left(e^{t / p} v\right)+e^{t / p} w \frac{\exp (-v)}{\int_{\Omega} \exp (-v) d x},
$$

defining $f(t, x):=\left(e^{t / p} v(t, x)-v_{0}(x)\right)$ we see that $f$ satisfies

$$
f_{t}=\Delta f-f+\frac{1}{p}\left(e^{t / p} v\right)+e^{t / p} w \frac{\exp (-v)}{\int_{\Omega} \exp (-v(s, x)) d x}+\Delta v_{0}-v_{0}
$$

as well as $f(0, x)=0$. Hence, we are in a position to apply the $L^{p}$ - $L^{q}$ maximal regularity estimate (see $[9$, Theorem 3.1, (3.8)]) and deduce that

$$
\begin{aligned}
\int_{0}^{t}\left\|f_{s}(s)\right\|_{L^{q}(\Omega)}^{p} d s \leq C_{\mathrm{MR}} & \left(t\left\|v_{0}\right\|_{W^{2, q}(\Omega)}^{p}+\int_{0}^{t} p^{-p} e^{s}\|v\|_{L^{q}(\Omega)}^{p} d s\right. \\
& \left.+\int_{0}^{t} e^{s}\left\|w(s) \frac{\exp (-v(s))}{\int_{\Omega} \exp (-v(s, x)) d x}\right\|_{L^{q}(\Omega)}^{p} d s\right) .
\end{aligned}
$$

Next we notice that

$$
f_{s}=\left(e^{s / p} v-v_{0}\right)_{s}=\frac{1}{p} e^{s / p} v+e^{s / p} v_{s}
$$

and consequently, taking the $p$ th power and integrating in time, by the triangle inequality we arrive at

$$
\int_{0}^{t} e^{s}\left\|v_{s}\right\|_{L^{q}(\Omega)}^{p} d s \leq C\left(\int_{0}^{t} e^{s}\left\|f_{s}\right\|_{L^{q}(\Omega)}^{p} d s+\int_{0}^{t} p^{-p} e^{s}\|v\|_{L^{q}(\Omega)}^{p} d s\right) .
$$

Plugging the above inequality in the left-hand side of (3.8), we obtain

$$
\begin{aligned}
& \int_{0}^{t} e^{s}\left\|v_{s}(s, x)\right\|_{L^{q}(\Omega)}^{p} d s \\
& \leq C\left(t\left\|v_{0}\right\|_{W^{2, q}(\Omega)}^{p}+\int_{0}^{t} e^{s}\left\|w(s) \frac{\exp (-v(s))}{\int_{\Omega} \exp (-v(s, x)) d x}\right\|_{L^{q}(\Omega)}^{p} d s\right. \\
& \left.\quad+p^{-p} \int_{0}^{t} e^{s}\|v(s)\|_{L^{q}(\Omega)}^{p} d s\right) \\
& \leq C\left(t\left\|v_{0}\right\|_{W^{2, q}(\Omega)}^{p}+H^{p} C\left(u_{0}, v_{0}\right)^{p} e^{t}+v^{*}(H)^{p}|\Omega|^{p / q} e^{t}\right),
\end{aligned}
$$


where in the last inequality we have used the fact that $\|w\|_{L^{\infty}(\Omega)} \leq H$ on $S_{2}(\tau)$ and $(3.4)$ in estimating the second term, while Lemma 3.5 has been used to bound the last term, so that the constant $v^{*}(H)$ appears. Multiplying the above inequality by $e^{-t}$, using the $L^{\infty}$ bound of $v$ on $S_{2}(\tau)$ again and noticing that

$$
\frac{\partial \log z}{\partial t}=-v(t) \frac{\partial v}{\partial t}
$$

we complete the proof.

By [6, Lemma 4.6] we obtain, for all $t \in\left(0, S_{2}(\tau)\right)$,

$$
\|w(t)\|_{L^{\infty}(\Omega)} \leq\|w(0)\|_{L^{\infty}(\Omega)}+C\|\nabla w(t)\|_{L^{q}(\Omega)} \quad \text { for } q>n,
$$

where $C$ is a positive constant depending on $H, v_{0}$ and $q$.

Proof of Theorem 3.2. The rest of the proof follows the lines of [6], but for the reader's convenience we give the details. First of all, notice that a crucial weighted Poincaré inequality in [6, Lemma 2.6] requires the weight (in our case $z_{j}$ ) to be bounded from above as well as to be bounded away from 0 . To ensure that, we notice that

$$
\frac{\|z\|_{L^{\infty}\left(\left(0, S_{2}(\tau)\right) \times \Omega\right)}}{\inf _{(t, x) \in\left(0, S_{2}(\tau)\right) \times \Omega} z(t, x)} \leq \frac{C\left(u_{0}, v_{0}\right) \exp \left(-v^{*}(H)\right)}{|\Omega|} .
$$

Then, by [6. Lemma 2.6],

$$
\Lambda^{*} \int_{\Omega}\left|\varphi-\frac{\int_{\Omega} z \varphi d x}{\int_{\Omega} z d x}\right|^{2} z(t) d x \leq \int_{\Omega}|\nabla \varphi|^{2} z(t) d x
$$

for $\varphi \in H^{1}(\Omega), t \in\left(0, S_{2}(\tau)\right)$ and $\tau \in(0,1]$. The above inequality plays a crucial role in the proofs of regularity estimates for $\mathcal{A}_{j}$ in [6, Lemma 2.8] which will be utilized further. We remark that the positive constant $\Lambda^{*}$ depends only on $n, \Omega, \min _{\bar{\Omega}} z, \max _{\bar{\Omega}} z$.

For $j=0,1, \ldots, J-1$ and $t \in\left(T_{j}, T_{j+1}\right)$ putting $\zeta=\left(t-T_{j}\right) \tau^{-1}$ and $W(\zeta, x)=w(t, x), Z(\zeta, x)=z(t, x), Z_{0}(x)=z_{j}(x)=z\left(T_{j}, x\right)$ and $Q(\zeta, x)=$ $\frac{\partial \log z}{\partial t}(t, x)$, we see that for $\zeta \in(0, T /(2 \tau))$,

$$
\frac{\partial W}{\partial \zeta}=\mathcal{A}_{j} W+\nabla \log \frac{Z}{Z_{0}} \cdot \nabla W-\tau Q W .
$$

Put

$$
F:=\nabla \log \frac{Z}{Z_{0}} \cdot \nabla W-\tau Q W .
$$

Then the function $W$ can be rewritten as

$$
W(\zeta)=e^{\zeta \mathcal{A}_{j}} W(0)+\int_{0}^{\zeta} e^{(\zeta-\xi) \mathcal{A}_{j}} F(\xi) d \xi .
$$


For $\zeta \in(0, T /(2 \tau))$, [6, Lemma 2.8(iii)] implies that

$$
\begin{aligned}
\|\nabla W(\zeta)\|_{L^{q}(\Omega)} & \leq\left\|\nabla e^{\zeta \mathcal{A}_{j}} W(0)\right\|_{L^{q}(\Omega)}+\int_{0}^{\zeta}\left\|\nabla e^{(\zeta-\xi) \mathcal{A}_{j}} F(\xi)\right\|_{L^{q}(\Omega)} d s \\
& \leq C e^{-\zeta \Lambda^{*}}\|\nabla W(0)\|_{L^{q}(\Omega)}+\mathbf{I}_{1}+\mathbf{I}_{2},
\end{aligned}
$$

where

$$
\begin{aligned}
& \mathbf{I}_{1}:=\int_{0}^{\zeta}\left\|\nabla e^{(\zeta-\xi) \mathcal{A}_{j}} \nabla \log \frac{Z(\xi)}{Z_{0}} \cdot \nabla W(\xi)\right\|_{L^{q}(\Omega)} d s, \\
& \mathbf{I}_{2}:=\tau \int_{0}^{\zeta}\left\|\nabla e^{(\zeta-\xi) \mathcal{A}_{j}} Q(\xi) W(\xi)\right\|_{L^{q}(\Omega)} d s .
\end{aligned}
$$

By [6, Lemma 2.8(i)], for $\mathcal{A}=\mathcal{A}(z)$ with a smooth positive function $z$ and for all $q \in(1, \infty)$, there exists some $C>0$ such that $\left\|\nabla e^{t \mathcal{A}} w_{0}\right\|_{L^{q}(\Omega)} \leq C_{3}\left(1+t^{-1 / 2}\right) e^{-\Lambda t}\left\|w_{0}\right\|_{L^{q}(\Omega)} \quad$ for $w_{0} \in L^{q}(\Omega)$ and $t>0$.

Notice that $\nabla Z(\zeta, x)=\nabla z(t, x)$ and hence we deduce from [6, Lemma 2.8(i)] and Lemma 3.6 that for $\zeta \in(0, T /(2 \tau))$,

$$
\begin{aligned}
\mathbf{I}_{1} & \leq C \int_{0}^{\zeta}\left\{1+\frac{1}{\sqrt{\zeta-\xi}}\right\} e^{(\xi-\zeta) \Lambda^{*}}\left\|\nabla \log \frac{z}{z_{j}}\right\|_{L^{\infty}(\Omega)}\|\nabla W\|_{L^{q}(\Omega)} d \xi \\
& \leq C\left(H, v_{0}\right) T^{\alpha / 2} \int_{0}^{\zeta}\left\{1+\frac{1}{\sqrt{\zeta-\xi}}\right\} e^{(\xi-\zeta) \Lambda^{*}}\|\nabla W\|_{L^{q}(\Omega)} d \xi \\
& \leq C\left(H, v_{0}\right) T^{\alpha / 2} \sup _{\xi \in[0, \zeta]}\|\nabla W(\xi)\|_{L^{q}(\Omega)}
\end{aligned}
$$

and that for $\zeta \in(0, T /(2 \tau))$,

$$
\mathbf{I}_{1} \leq C\left(H, v_{0}\right) T^{\alpha / 2} e^{-\zeta \Lambda^{*}} \sup _{\xi \in[0, T /(2 \tau)]} e^{\xi \Lambda^{*}}\|\nabla W(\xi)\|_{L^{q}(\Omega)} .
$$

Exactly as in [6, pp. 1665-1666] (Lemma 3.7 is required here!), we arrive at

$$
\mathbf{I}_{2} \leq C\left(H, v_{0}\right) \tau^{n /(n+1)} .
$$

Put $B_{0}=\left\|\nabla w_{0}\right\|_{L^{n+1}(\Omega)}$ and

$$
B=\sup _{\xi \in[0, T /(2 \tau)]} e^{\xi \Lambda^{*}}\|\nabla W(\xi)\|_{L^{n+1}(\Omega)}=\sup _{t \in\left[T_{j}, T_{j+1}\right]} e^{\left(t-T_{j}\right) \Lambda^{*} / \tau}\|\nabla w(t)\|_{L^{n+1}(\Omega)} .
$$

By (3.11), 3.13 and 3.14, there exists a positive constant $C\left(H, v_{0}\right)$ such that

$$
B \leq C\left(H, v_{0}\right) B_{0}+C\left(H, v_{0}\right) T^{\alpha / 2} B+C\left(H, v_{0}\right) \tau^{n /(n+1)} e^{T \Lambda^{*} /(2 \tau)},
$$


so that, by taking $\tau$ and $T$ small enough to ensure

$$
C\left(H, v_{0}\right) T^{\alpha / 2} \leq 1 / 2, \quad C\left(H, v_{0}\right) \tau^{n /(n+1)} \leq \tau^{1 / 3} / 8,
$$

we obtain

$$
B \leq 2 C\left(H, v_{0}\right) B_{0}+\frac{1}{4} e^{T \Lambda^{*} /(2 \tau)} \tau^{1 / 3} .
$$

Next, we choose $\tau$ small enough to have

$$
C\left(H, v_{0}\right) e^{-T \Lambda^{*} /(2 \tau)} \leq \tau / 8 .
$$

In view of this choice, owing to 3.15$)$, we arrive at

$$
\begin{aligned}
\|\nabla W(T /(2 \tau))\|_{L^{n+1}(\Omega)} & \leq 2 C\left(H, v_{0}\right) e^{-T \Lambda^{*} /(2 \tau)}\|\nabla W(0)\|_{L^{n+1}(\Omega)}+\frac{1}{4} \tau^{1 / 3} \\
& \leq \frac{\tau}{4}\|\nabla W(0)\|_{L^{n+1}(\Omega)}+\frac{\tau^{1 / 3}}{4} .
\end{aligned}
$$

Since $W(T /(2 \tau))=w\left(T_{j+1}\right)$ and $W(0)=w\left(T_{j}\right)$, we see that

$$
\left\|\nabla w\left(T_{j+1}\right)\right\|_{L^{n+1}(\Omega)} \leq \frac{\tau}{4}\left\|\nabla w\left(T_{j}\right)\right\|_{L^{n+1}(\Omega)}+\frac{\tau^{1 / 3}}{4} \quad \text { for } j=0,1, \ldots, J-1,
$$

and that

$$
\begin{aligned}
& \left\|\nabla w\left(T_{j}\right)\right\|_{L^{n+1}(\Omega)} \leq \frac{\tau}{4}\left\|\nabla w\left(T_{j-1}\right)\right\|_{L^{n+1}(\Omega)}+\frac{\tau^{1 / 3}}{4} \\
& \leq\left(\frac{\tau}{4}\right)^{j}\left\|\nabla w_{0}\right\|_{L^{n+1}(\Omega)}+\frac{\tau^{1 / 3}}{4}\left\{1+\frac{\tau}{4}+\left(\frac{\tau}{4}\right)^{2}+\cdots+\left(\frac{\tau}{4}\right)^{j-1}\right\} \\
& \leq\left(\frac{\tau}{4}\right)^{j}\left\|\nabla w_{0}\right\|_{L^{n+1}(\Omega)}+\frac{\tau^{1 / 3}}{2} \quad \text { for } j=0,1, \ldots, J .
\end{aligned}
$$

Combining this with (3.11), (3.12) and (3.14), keeping the previous choice of $\tau$, we infer that

$$
\begin{aligned}
& \sup _{\xi \in[0, T /(2 \tau)]}\|\nabla W(\xi)\|_{L^{n+1}(\Omega)} \\
& \leq C\left(H, v_{0}\right)\|\nabla W(0)\|_{L^{n+1}(\Omega)}+C\left(H, v_{0}\right) T^{\alpha / 2} \sup _{\xi \in[0, T /(2 \tau)]}\|\nabla W(\xi)\|_{L^{n+1}(\Omega)} \\
&+C\left(H, v_{0}\right) \tau^{n /(n+1)} \\
& \leq C\left(H, v_{0}\right)\left\{\left(\frac{\tau}{4}\right)^{j}\left\|\nabla w_{0}\right\|_{L^{n+1}(\Omega)}+\frac{\tau^{1 / 3}}{2}\right\} \\
&+\frac{1}{2} \sup _{\xi \in[0, T /(2 \tau)]}\|\nabla W(\xi)\|_{L^{n+1}(\Omega)}+\frac{\tau^{1 / 3}}{8} .
\end{aligned}
$$

Finally, in addition to the condition on $\tau$ we have assumed so far, we also require that $\tau$ is small enough that

$$
2 C\left(H, v_{0}\right)\left\{\frac{\tau}{4}\left\|\nabla w_{0}\right\|_{L^{n+1}(\Omega)}+\frac{\tau^{1 / 3}}{2}\right\}+\frac{\tau^{1 / 3}}{4} \leq \tau^{1 / 6} .
$$


This allows us to conclude that

$$
\|\nabla w(t)\|_{L^{n+1}(\Omega)} \leq C \tau^{1 / 6} \quad \text { for } t \in\left[T / 2, S_{2}(\tau)\right],
$$

where $C$ is a positive constant depending on $H, v_{0}$ and $u_{0}$. In view of 3.9 we obtain

$$
\|w(t)\|_{L^{\infty}(\Omega)} \leq\|w(0)\|_{L^{\infty}(\Omega)}+C \tau^{1 / 6} \quad \text { for } t \in\left[T / 2, S_{2}(\tau)\right],
$$

which allows us, by taking $\tau$ small and due to the definitions of $T$ and $H$, to arrive at

$$
\|w(t)\|_{L^{\infty}(\Omega)}<H \quad \text { for } t \in\left[0, S_{2}(\tau)\right] .
$$

But the above inequality contradicts $S_{2}(\tau)<\infty$. This means that $T_{\max }=$ $S_{2}(\tau)=\infty$. So, we deduce the time global boundedness of $(w, v)$, which eventually gives boundedness of $(u, v)$. The proof of Theorem 3.2 is complete.

Acknowledgements. T. C. was supported by the Polish National Science Centre under grant SONATA BIS 7 number UMO-2017/26/E/ST1/ 00989. K. F. is supported by Grant-in-Aid for Early-Career Scientists (No. 19K14576), Japan Society for the Promotion of Science.

\section{References}

[1] D. Bakry, I. Gentil and M. Ledoux, Analysis and Geometry of Markov Diffusion Operators, Springer, 2014.

[2] D. Bakry and M. Ledoux, A logarithmic Sobolev form of the Li-Yau parabolic inequality, Rev. Mat. Iberoamer. 22 (2006), 683-702.

[3] D. Bothe, A. Fischer, M. Pierre and G. Rolland, Global existence for diffusionelectromigration systems in space dimension three and higher, Nonlinear Anal. 99 (2014), 152-166.

[4] T. Cieślak, Ph. Laurençot and C. Morales-Rodrigo, Global existence and convergence to steady states in a chemorepulsion system, in: Parabolic and Navier-Stokes Equations, Banach Center Publ. 81, Inst. Math., Polish Acad. Sci., 2008, 105-117.

[5] M. Freitag, Global existence and boundedness in a chemorepulsion system with superlinear diffusion, Discrete Contin. Dynam. Systems 38 (2018), 5943-5961.

[6] K. Fujie and T. Senba, A sufficient condition of sensitivity functions for boundedness of solutions to a parabolic-parabolic chemotaxis system, Nonlinearity 31 (2018), 16391672 .

[7] K. Fujie and T. Senba, Application of an Adams type inequality to a two-chemical substances chemotaxis system, J. Differential Equations 263 (2017), 88-148.

[8] R. Hamilton, A matrix Harnack estimate for the heat equation, Comm. Anal. Geom. 1 (1993), 113-126.

[9] M. Hieber and J. Prüss, Heat kernels and maximal $L^{p}-L^{q}$ estimates for parabolic evolution equations, Comm. Partial Differential Equations 22 (1997), 1647-1669.

[10] P. Li and S.-T. Yau, On the parabolic kernel of the Schrödinger operator, Acta Math. 156 (1986), 153-201.

[11] K. Lin and C. Mu, Global existence and convergence to steady states for an attractionrepulsion chemotaxis system, Nonlinear Anal. Real World Appl. 31 (2016), 630-642. 
[12] M. S. Mock, An initial value problem from semiconductor device theory, SIAM J. Math. Anal. 5 (1974), 597-612.

[13] Y. Tao, Global dynamics in a higher-dimensional repulsion chemotaxis model with nonlinear sensitivity, Discrete Contin. Dynam. Systems Ser. B 18 (2013), 2705-2722.

[14] Y. Tao and Z.-A. Wang, Competing effects of attraction vs. repulsion in chemotaxis, Math. Models Methods Appl. Sci. 23 (2013), 1-36.

Tomasz Cieślak Institute of Mathematics Polish Academy of Sciences 00-656 Warszawa, Poland E-mail: cieslak@impan.pl
Kentarou Fujie Research Alliance Center for Mathematical Sciences Tohoku University Sendai, 980-8578, Japan E-mail: fujie@tohoku.ac.jp 